\title{
ON OSCULATING ELEMENT-BANDS ASSOCIATED WITH LOCI OF
}

\section{SURFACE-ELEMENTS*}

\author{
BY

\section{PERCEY F. SMITH}

In a memoir by ScheFFers, entitled Isogonalcurven, Aequitangentialcurven und complexe Zahlen, which appeared in volume 60 of the Mathematische Annalen (1905), p. 491, a number of theorems were demonstrated concerning properties of the osculating circles of certain loci of line-elements in the plane. Two of these may be cited here. Given a differential equation of the first order,

$$
f(x, y, p)=0,
$$

in which $p=d y / d x$, and the one-parameter group

$$
x=x, \quad y=y, \quad \arctan p=\arctan p^{\prime}+t,
$$

by which each line-element is turned about its point through the same angle, then the transformed elements satisfy the equation

$$
f\left(x, y, \frac{p^{\prime}+\tan \tau}{1-p^{\prime} \tan \tau}\right)=0,
$$

the integral curves of which are isogonal trajectories of the integral curves of the original equation (1). The first theorem in question is now this: The osculating circles of all the isogonal trajectories through a fixed point pass also through a second fixed point, that is, form a pencil. Furthermore, these circles will osculate at the second point another system of isogonal trajectories.

The second theorem is similar in character if the group (2) is replaced by

$$
x=\bar{x}+\frac{t}{\sqrt{1+p^{2}}}, \quad y=\bar{y}+\frac{p t}{\sqrt{1+p^{2}}}, \quad p=p,
$$

which has the effect of sliding each line-element along its line the same distance $t$. The integral curves of the transformed equation

$$
f\left(\bar{x}+\frac{t}{\sqrt{1+p^{2}}}, \quad \bar{y}+\frac{p t}{\sqrt{1+p^{2}}}, \quad p\right)=0
$$

are called by Scheffers aequitangential curves of the original system (1). Then

\footnotetext{
* Presented to the Society, September 15, 1909, and February 26, 1910.
} 
the theorem holds: The osculating circles of the aequitangential curves which touch a given line will touch also a second line. Furthermore, these circles will osculate on the second line a second system of aequitangential curves.

The discussion of the present paper leads to similar theorems for loci of surface-elements in space. Such loci are regarded as associations of element-bands, and either of two species of simple bands - parabolic and cubic - appear in the same role as osculating circles in the plane. The investigation is much simplified by means of transformations which make the problem depend upon projective geometry in space of five dimensions. This method has been employed to advantage by EIEsLAND in a paper referred to below.

As far as the author is aware, the question of osculating element-bands is discussed for the first time in this paper.

\section{§1. Preliminary Theorems.}

Two united line-elements in the plane determine a unique curvature-element $\left(x, y, y^{\prime}, y^{\prime \prime}\right)$. For, given two such elements $\left(x, y, y^{\prime}\right)$ and $(x+d x, y+d y$, $\left.y^{\prime}+d y^{\prime}\right)$, where $d y=y^{\prime} d x$, then if $y^{\prime \prime}$ is defined by the equation $d y^{\prime}=y^{\prime \prime} d x$, it is clear that the ratios $d x: d y: d y^{\prime}$ determine $y^{\prime \prime}$ uniquely.

The case is different, however, with two united surface-elements in space. For convenience we may adopt the notation $(P, E)$ for such an element, $P$ being the point and $E$ the plane of the element. Assuming two united elements $(P, E)$ and $\left(P^{\prime}, E^{\prime}\right)$ whose coördinates are $(x, y, z, p, q)$ and $(x+d x, y+d y, z+d z, p+d p, q+d q)$, where $d z=p d x+q d y$, then of the ratios

$$
\frac{d x}{\alpha}=\frac{d y}{\beta}=\frac{d z}{p \alpha+q \beta}=\frac{d p}{\delta}=\frac{d q}{\epsilon},
$$

three are independent. On introducing the numbers $r, s$ and $t$ defined by the equations

or also, from (1),

$$
d p=r d x+s d y, \quad d q=s d x+t d y
$$

$$
\delta=r \alpha+s \beta, \quad \epsilon=s \alpha+t \beta,
$$

it is obvious that $r, s, t$ are not determined uniquely when the ratios (1) are given, but that, in fact, we have the result: * Two united surface-elements in space determine $\infty^{1}$ curvature-elements $(x, y, z, p, q, r, s, t)$.

Consider now any surface to which the united elements under discussion belong. The curvature of normal sections at $P$ is, by the well-known formula, given by

$$
\frac{1}{R}=\frac{1}{\sqrt{1+p^{2}+q^{2}}} \frac{r d x^{2}+2 s d x d y+t d y^{2}}{d s^{2}},
$$

* EngkL, Die hoheren Differentialquotienten, Leipziger Berichte (1893), p. 475. 
where $d s$ is the linear element. If we substitute $r$ and $t$ in terms of $s$ from (3), then (4) becomes

$$
\frac{1}{R}=\frac{1}{\sqrt{1+p^{2}+q^{2}}} \cdot \frac{\beta \delta d x^{2}+\alpha \epsilon d y^{2}-s(\beta d x-\alpha d y)^{2}}{\alpha \beta d s^{2}},
$$

in which of course, for any given value of $d x: d y, s$ is arbitrary. We observe, however, that the curvature is the same for all values of $s$ when the normal section is such that $\beta d x-a d y=0$, that is, such that (1) is satisfied. The result may be expressed as follows: The $\infty^{1}$ curvature-elements determined by two united surface-clements $(P, E)$ and $\left(P^{\prime}, E^{\prime}\right)$ hang together along the direction $P P^{\prime}$ in such a manner that the curvature at $P$ of all normal sections containing $P P^{\prime}$ is the same.

From equations (3), we readily find that

$$
\alpha \beta\left(r t-s^{2}\right)=-(\alpha \delta+\beta \epsilon) s+\delta \epsilon .
$$

If, then, $\alpha \delta+\beta \epsilon \neq 0$, one of the curvature-elements has zero total curvature. On the other hand, when $\alpha \delta+\beta \epsilon=0$, the total curvature of all the $\infty^{1}$ curvature-elements is the same.

Finally, denoting by $\delta x: \delta y$ the direction conjugate to $d x: d y$ on any surface containing the pair of elements under consideration, then the usual formula $r d x \hat{\delta} x+s(d x \delta y+d y \delta x)+t d y \delta y=0$ gives by substitution from (3), and on solving for $\delta x: \delta y$,

$$
\frac{\delta x}{\delta y}=-\frac{\alpha}{\beta} \frac{\epsilon d y+s(\beta d x-\alpha d y)}{\delta d x-s(\beta d x-\alpha d y)} .
$$

Hence if $\beta d x-\alpha d y=0$, the conjugate direction is the same for all values of $s$, namely, $\delta \delta x+\epsilon \delta y=0$. In words: On all surfaces containing two united surface-elements $(P, E)$ and $\left(P^{\prime}, E^{\prime}\right)$ the direction conjugate to $P P^{\prime}$ is the same.

This fact is obvious geometrically. For this common conjugate direction is determined by the line of intersection of the planes $E$ and $E^{\prime}$ of the united elements.

Evidently, if $\alpha \delta+\beta \epsilon=0$, the conjugate direction is identical with $P P^{\prime}$, and the latter direction is therefore an asymptotic direction. This fact when combined with the conclusion found in discussing (6) gives the result: If the total curvature of the $\infty^{1}$ curvature-elements (3) is the same, the direction along which they are united is an asymptotic direction.

\section{§2. Parabolic and Cubic Bands.}

In the Differential Geometry of the plane, the circle plays a conspicuous rôle. This fact, when metric properties are left out of consideration, is explained by remarking that a circle is uniquely determined by two of its united elements. Plainly, a curve of any family depending upon three parameters will have this 
last property - vertical parabolas, for example. The point is that we consider unions of line-elements which are determined when two united elements are given.

An analogous problem for space now arises. We wish to consider unions of $\infty^{1}$ surface-elements each one of which is determined if two united elements are given. Let the term element-band be used for a union of $\infty^{1}$ surface-elements. Analytically an element-band is defined by
$x=x(t)$,
$y=y(t)$
$z=z(t)$,
$p=p(t)$
$q=q(t)$

provided that $z^{\prime}=p x^{\prime}+q y^{\prime}$, where accents indicate derivatives with respect to the parameter $t$.

Two species of element-bands, now to be described, appear in this paper. The points of the elements lie on a curve, which may be called the point-locus, while the planes form a developable, called the plane-locus. The two species of element-bands may be respectively named parabolic bands and cubic bands.

The point-locus of a parabolic band is a parabola whose axis is parallel to the $Z$-axis. The plane-locus is a parabolic cylinder. To conceive such a band we may think of such a parabola, which may be called a vertical parabola then imagine a line meeting the parabola to generate a cylinder, and finally picture to ourselves a narrow band of the cylindrical surface along the parabola. Clearly, $\infty^{2}$ parabolic bands may have a common point-locus or dually, the same plane-locus. To dwell for a moment on the last point: - the parabolic cylinders in question obviously contain the point at infinity on the Z-axis. Given such a cylinder, then any plane through the point referred to determines on the cylinder a parabolic band.

The point-locus of a cubic band is a skew cubic. The plane-locus is a quadric cone whose vertex lies on the cubic. Clearly there are $\infty^{1}$ cubic bands with a common point-locus. On the other hand, the multiplicity of the system of cubic bands belonging to a quadric cone is the same as that of the system of skew cubics lying on it, and is of no special importance at this moment. The concept of a cubic band is readily visualized if we think of a skew cubic, picture any quadric cone standing on it and think of a narrow band of the conical surface along the cubic.

The fact is now readily established that a parabolic band is determined by two of its united elements, $(P, E)$ and $\left(P^{\prime}, E^{\prime}\right)$. For pass through $P P^{\prime}$ the plane parallel to the Z-axis. The united line-elements in this plane determined by its intersection with $E$ and $E^{\prime}$ determine a vertical parabola. Further, the line of intersection of $E$ and $E^{\prime}$ gives an element of the parabolic cylinder. The multiplicity of all parabolic bands is obviously seven-fold. For there are $\infty^{8}$ pairs of united elements and $\infty^{1}$ elements determine the same parabolic band. The important exceptional case already noted - when $E$ and $E^{\prime}$ intersoct along $P P^{\prime}$ - leads to a twisted band. Namely, the point-lorus is now the 
line $P P^{\prime}$ and the planes of the plane-locus pass through $P P^{\prime}$. The points and planes of the elements of the band are in a projective relation, such that $P$ and $E, P^{\prime}$ and $E^{\prime}$ are corresponding elements, and moreover, the point at infinity on $P P^{\prime}$ corresponds to the vertical plane through $P P^{\prime}$.

Turn next to the question of the determination of a cubic band by a pair of united surface-elements. From the results of $\S 1$, it is clear that the vertex of any cone to which the united elements $(P, E)$ and $\left(P^{\prime}, E^{\prime}\right)$ belong, must lie on the line of intersection of $E$ and $E^{\prime}$. For this direction is conjugate to $P P^{\prime}$, and for the present we assume these directions are not identical. Denote the line of intersection of $E$ and $E^{\prime}$ by $\left(E, E^{\prime}\right)$. Let us now confine ourselves to quadric cones. Consider the normal section of such a cone through the direction $P P^{\prime}$. Then by $\S 1$, the curvature of this conic at $P$ is given. Consequently three conditions are imposed upon this conic. It is therefore clear that $\infty^{3}$ quadric cones may contain the same pair of united elements. The cones touch along a common element $\left(E, E^{\prime}\right)$ and in addition, their normal sections through $P P^{\prime}$ osculate at $P$. To obtain a unique cone of this system we may introduce the three fixed conditions - the cones shall pass through three non-collinear fixed points $A, B$ and $C$. It is easy to see that the required cone is now uniquely determined. For, in the first place, its trace $c^{\prime}$ on the plane of $A, B$ and $C$ is determined. Secondly, the normal section through $P P^{\prime}$ now has two points given on this trace $c^{\prime}$, and hence is a unique conic $c^{\prime \prime}$. Only one cone with a given element can contain two conics.

We see, therefore, that a pair of united surface-elements $(P, E),\left(P^{\prime}, E^{\prime}\right)$ will determine a unique quadric cone $K$ standing on three fixed points $A, B$, $C$. Let the vertex of this cone be $V$. Consider the quadric cone whose vertex is $A$ which stands on the five points $P, P^{\prime}, V, B$ and $C$. Its intersection with the first cone is a skew cubic $C_{3}$ and the element $V A$. Similarly each of the fixed points $B$ and $C$ is the vertex of a quadric cone which will contain the same cubic. The cubic band lying on $K$ along $C_{3}$ contains $(P, E)$ and $\left(P^{\prime}, E^{\prime}\right)$ and furthermore is uniquely determined by them.

The multiplicity of skew cubics passing through three non-collinear fixed points is six-fold. There are accordingly $\infty^{7}$ cubic bands, $\infty^{1}$ on each of the skew cubics of the system. On the other hand, on each quadric cone standing on the fixed points $A, B$ and $C$ lie $\infty^{2}$ cubic bands, and the multiplicity of such cones is five-fold.

If the line of intersection of the planes $E$ and $E^{\prime}$ is identical with $P P^{\prime}$, the cubic band is constructed by assuming $E$ as the osculating plane at $P$, and $P P^{\prime}$, of course, as the tangent. The cubic satisfying this condition and moreover passing through $A, B$, and $C$ is unique. The cone $K$ then has its vertex at $P$. 


\section{§3. Osculating Bands.}

Two element-bands having in common two united elements may be said to osculate. The analytical conditions are easily derived. For given two bands

and

$$
x=f(t), \quad y=g(t), \quad z=h(t), \quad p=\phi(t), \quad q=\chi(t),
$$

$$
x=f_{1}(t), \quad y=g_{1}(t), \quad z=h_{1}(t), \quad p=\phi_{1}(t), \quad q=\chi_{1}(t),
$$

then osculation demands the equality of $f$ and $f_{1}, g$ and $g_{1}$, etc., and their first derivatives for some value of $t$. In these conditions, however, the relation $h^{\prime}=h_{1}^{\prime}$ depends upon the others from the conditions for bands, (1), § 2 .

Geometrically, two element-bands osculate when their point-loci touch and their plane-loci are tangent along a common generator.

The immediate subject of investigation concerns properties of surface-element loci with respect to osculating parabolic or cubic bands. It is clear that there are $\infty^{1}$ bands of this character associated with any element-band. Further, given any surface or union of $\infty^{2}$ elements, then $\infty^{3}$ osculating parabolic or cubic bands are determined. Of particular interest, however, is the case when the surface-element locus is defined by a partial differential equation of the first order

$$
f(x, y, z, p, q)=0 .
$$

The $\infty^{4}$ elements satisfying (1) are arranged on $\infty^{3}$ bands, the characteristic bands. If the coördinates of two united elements of a characteristic band are $(x, y, z, p, q)$ and $(x+d x, y+d y, z+d z, p+d p, q+d q)$, then these coördinates of course satisfy $f=0$ and further the well-known equations due to Lie,

$$
\frac{d x}{f_{p}}=\frac{d y}{f_{q}}=\frac{d z}{p f_{p}+q f_{q}}=\frac{d p}{-f_{x}-p f_{z}}=\frac{d q}{-f_{y}-q f_{z}} .
$$

Each element satisfying (1) is associated with a united element determined by (2) such that the pair of united elements lie on a characteristic band. Now, clearly, the parabolic or cubic band determined by this pair of elements will osculate the characteristic band. Thus at each element of the locus (1), there is determined a parabolic or cubic band which osculates the characteristic band containing the given element. There are $\infty^{4}$ osculating parabolic or cubic bands. If, now, we look upon the locus of (1) not as a set of integral surfaces, but as an association of $\infty^{3}$ element-bands, the characteristic bands, then the properties of osculating parabolic or cubic bands appear a natural subject for investigation. The $\infty^{4}$ parabolic or cubic bands. which osculate the characteristic bands of a given differential equation of the first order we shall refer to briefly as the osculating parabolic or cubic bands of the locus of the equation. 


\section{§4. Transformation of the Problem.}

The investigation of the question proposed in the preceding section is greatly facilitated by the aid of transformations by which the geometry of surface-elements is made to depend upon projective geometry in a space of five dimensions $R_{5}$. Such a transformation has been given by Lre.* A second transformation is exhibited in $\S 7$ of this paper. The capital point is that parabolic or cubic bands correspond by these respective transformations to straight lines in $R_{5}$. To disclose the details, we begin by examining the transformation already referred to as due to Lie. This is defined by the equations

(1) $\quad X_{1}=x, \quad X_{2}=y, \quad Y_{1}=\frac{1}{2} p, \quad Y_{2}=\frac{1}{2} q, \quad Z=z-\frac{1}{2} p x-\frac{1}{2} q y$,

by which identically

$$
d Z+X_{1} d Y_{1}-Y_{1} d X_{1}+X_{2} d Y_{2}-Y_{2} d X_{2}=d z-p d x-q d y .
$$

If we interpret $\left(X_{1}, X_{2}, Y_{1}, Y_{2}, Z\right)$ as cartesian point coördinates in $R_{5}$, then clearly united surface-elements in ordinary space $R_{3}$ transform into lineelements in $R_{5}$ satisfying the equation

$$
d Z+X_{1} d Y_{1}-Y_{1} d X_{1}+X_{2} d Y_{2}-Y_{2} d X_{2}=0 .
$$

Now equation (3) is the differential equation of the null-system, $\uparrow$

$$
Z-Z^{\prime}+Y_{1} X_{1}^{\prime}-X_{1} Y_{1}^{\prime}+Y_{2} X_{2}^{\prime}-X_{2} Y_{2}^{\prime}=0,
$$

that is, of a correlation in $R_{5}$ such that point and corresponding linear $M_{4}$ are incident.

A line in $R_{5}$,

(5) $X_{1}=X_{1}^{0}+\lambda_{1} t, X_{2}=X_{2}^{0}+\lambda_{2} t, Y_{1}=Y_{1}^{0}+\mu_{1} t, Y_{2}=Y_{2}^{0}+\mu_{2} t, Z=Z^{0}+\nu t$, whose elements satisfy (3), that is, such that

$$
\nu+\mu_{1} X_{1}-\lambda_{1} Y_{1}+\mu_{2} X_{2}-\lambda_{2} Y_{2}=0,
$$

is a null-line. There are plainly $\infty^{7}$ null-lines.

By this transformation (1), the null-line in $R_{5}$ becomes in $R_{3}$ an element-band. This is a parabolic band, as shall now appear. Transforming (5), we obtain for the equations of the corresponding band, using $\left(5^{\prime}\right)$,

$$
\begin{gathered}
x=x^{0}+\lambda_{1} t, \quad y=y^{0}+\lambda_{2} t, \quad z=z^{0}+\left(\lambda_{1} p^{0}+\lambda_{2} q^{0}\right) t+\left(\lambda_{1} \mu_{1}+\lambda_{2} \mu_{2}\right) t^{2}, \\
p=p^{0}+2 \mu_{1} t, \quad q=q^{0}+2 \mu_{2} t .
\end{gathered}
$$

The point-locus of this band is, in the general case, a vertical parabola. $\neq$ The

* LiE-EngeL, Transformationsgruppen, vol. 2, p. 521.

$\dagger$ For details, see Eresland, On Null-Systems in Space of Five Dimensions, eto., American Journal of Mathematics, vol. 26 (1904), p. 103.

$\ddagger$ Eiesland, 1. c., p. 120. 
plane-locus is the envelope of

$$
z^{\prime}-z=p\left(x^{\prime}-x\right)+q\left(y^{\prime}-y\right),
$$

in which $x, y, z, p, q$, have the values in (6). On substituting their values in (7), the equation takes the form

$$
\left(\lambda_{1} \mu_{1}+\lambda_{2} \mu_{2}\right) t^{2}-2\left[\mu_{1}\left(x^{\prime}-x^{0}\right)+\mu_{2}\left(y^{\prime}-y^{0}\right)\right] t+z^{\prime}-z^{0}-p^{0}\left(x^{\prime}-x^{0}\right)-q^{0}\left(y^{\prime}-y^{0}\right)=0 .
$$

The envelope of (8) is clearly, in the general case, a parabolic cylinder. The band (6) is accordingly a parabolic band. Certain exceptional cases are necessary for the following discussion.

1. If $\lambda_{1}=\lambda_{2}=0$, then (6) becomes $x=x^{0}, y=y^{0}, z=z^{0}$. That is, the point-locus is a point. The $\infty^{1}$ surface-elements now form a hinge, for the planes (8) form a pencil.

2. If $\lambda_{1} \mu_{1}+\lambda_{2} \mu_{2}=0$ and $\lambda_{1} \neq 0, \lambda_{2} \neq 0$, the point-locus is a line and the plane-locus a plane-pencil. The band is now a twisted band, the points and planes of the element forming a projective series.

3. If $\mu_{1}=\mu_{2}=0$, the band just discussed becomes flat, that is, a straight band on a plane.

It is clear from these results that the contact geometry of parabolic bands in $R_{3}$ becomes under the transformation (1) the projective geometry of a null-system. The group of the latter is easily found to depend upon 21 parameters.*

In later sections it will be shown that the null-lines (5) are by another transformation changed into cubic bands, and finally that parabolic and cubic bands correspond under a contact transformation.

\section{§5. Theorems on the Null-System in $R_{5}$.}

Certain theorems regarding the null-system

$$
Z-Z^{\prime}+Y_{1} X_{1}^{\prime}-X_{1} Y_{1}^{\prime}+Y_{2} X_{2}^{\prime}-X_{2} Y_{2}^{\prime}=0,
$$

necessary in the sequel, are discussed in this section.

The plane in $R_{5}$

$$
Y_{1}=a_{1} X_{1}+a_{2} X_{2}+a_{3}, \quad Y_{2}=b_{1} X_{1}+b_{2} X_{2}+b_{3}, \quad Z=c_{1} X_{1}+c_{2} X_{2}+c_{3}
$$

will contain the null-line $(5), \S 4$, if in addition to the obvious conditions that the point ( $X_{1}^{0}, X_{2}^{0}$, etc.) lies on (2), we have

$$
\mu_{1}=a_{1} \lambda_{1}+a_{2} \lambda_{2}, \quad \mu_{2}=b_{1} \lambda_{1}+b_{2} \lambda_{2}, \quad \nu=c_{1} \lambda_{1}+c_{2} \lambda_{2} .
$$

On substituting in the condition $\left(5^{\prime}\right), \S 4$, for a null-line, the single condition is obtained

(4) $\left[c_{1}-a_{3}+\left(b_{1}-a_{2}\right) X_{2}^{0}\right] \lambda_{1}+\left[c_{2}-b_{3}+\left(a_{2}-b_{1}\right) X_{1}^{0}\right] \lambda_{2}=0$.

* Eirgland, l. c., p. 142. 
The following conclusions are now apparent :

1. Through an arbitrary point in an arbitrary plane in $R_{5}$ there is one nullline.

2. All lines in the plane (2) through the point determined by

$$
X_{1}^{0}=\frac{b_{3}-c_{2}}{a_{2}-b_{1}}, \quad X_{2}^{0}=\frac{c_{1}-a_{3}}{a_{2}-b_{1}}
$$

are null-lines. This point is the null-point of the plane.

3. If $a_{2}=b_{1}, b_{3}=c_{2}, c_{1}=a_{3}$, then every line in the plane is a null-line. The plane (2) is now a null-plane. The conditions obviously demand symmetry in the determinant of the right-hand members in (2). Explicitly, the equations (2) take the form, if the plane is a null-plane,

(6) $Y_{1}=a_{1} X_{1}+a_{2} X_{2}+a_{3}, \quad Y_{2}=a_{2} X_{1}+b_{2} X_{2}+c_{2}, \quad Z=a_{3} X_{1}+c_{2} X_{2}+c_{3}$.

If we next inquire how many null-planes contain a given null-line, the answer is immediate, namely, each null-line is the axis of a pencil of $\infty^{1}$ null-planes.

Clearly, all planes in $R_{5}$ cannot be represented in the form (2). The two exceptional cases are, for null-planes, given by

(8) $\quad X_{1}=a X_{2}+b, \quad Y_{2}=c X_{2}-a Y_{1}+e, \quad Z=e X_{2}-b Y_{1}+h$.

We may, in general, define a null-surface* in $R_{5}$ as one whose tangent planes are null-planes.

Turning now to the locus of one equation

$$
F\left(X_{1}, X_{2}, Y_{1}, Y_{2}, Z\right)=0 \text {, }
$$

defining in $R_{5}$ a manifoldness $M_{4}$ of four dimensions, the linear tangent $M_{4}^{1}$ is given as usual by

$$
\begin{aligned}
F_{X_{1}}\left(X_{1}^{\prime}-X_{1}\right)+F_{X_{2}}\left(X_{2}^{\prime}-X_{2}\right) & +F_{Y_{1}}\left(Y_{1}^{\prime}-Y_{1}\right) \\
& +F_{Y_{9}}\left(Y_{2}^{\prime}-Y_{2}\right)+F_{Z}\left(Z^{\prime}-Z\right)=0 .
\end{aligned}
$$

The null-plane (6) will pass through the point of contact $\left(X_{1}, X_{2}\right.$, etc.) and lie entirely within the locus of $(10)$ under these conditions:

$$
\begin{aligned}
& a_{3}=Y_{1}-a_{1} X_{1}-a_{2} X_{2}, \quad c_{2}=Y_{2}-a_{2} X_{1}-b_{2} X_{2}, \quad c_{3}=Z-a_{3} X_{1}-c_{2} X_{2}, \\
& F_{X_{1}}+a_{1} F_{Y_{1}}+a_{2} F_{Y_{2}}+a_{3} F_{Z}=0, \quad F_{X_{2}}+a_{2} F_{Y_{1}}+b_{2} F_{Y_{3}}+c_{2} F_{Z}=0 .
\end{aligned}
$$

There is no difficulty in showing that the $\infty^{1}$ null-planes satisfying (11) and (12) form a pencil whose axis is the null-line for which

$$
\frac{d X_{1}}{F_{Y_{1}}-\bar{X}_{1} F_{Z}^{-}}=\frac{d X_{2}}{F_{Y_{3}}-\bar{X}_{2} F_{Z}}=\frac{d Y_{1}}{-F_{X_{1}}-Y_{1} F_{Z}}=\frac{d Y_{2}}{-F_{Y_{3}}-Y_{2} F_{Z}}=\frac{d Z}{\Sigma X F_{X}^{\prime}},
$$

\footnotetext{
* For a development of this idea, see Ermsland, 1. c., p. 128.
} 
the denominator $\Sigma X F_{X}$ containing the four terms corresponding to $X_{1}, X_{2}$, $Y_{1}, Y_{2}$. We may express this important result as follows:

At each point of an $M_{4}$ in $R_{5}$ there is a pencil of tangent null-planes whose axis is the null-line (13).

The null- $M_{4}^{1}$ of $P$ contains every null-plane through $P$. Hence the result just found may be.formulated thus :

The tangent $M_{4}^{1}$ and null-M $M_{4}^{1}$ of $P$ intersect in a pencil of null-planes.

We shall call the axis of this pencil the tangent null-axis at $P$.

The result just stated is fundamental for the sequel. It is clear that the tangent null-axis at any point on the locus of (9) is distinguished from all other tangent null-lines, of which there are of course $\infty^{2}$.

Returning to the plane (2), we may represent this for the moment by the symbol $(a b c)$ of the determinant on the right-hand members. As already pointed out, if $(a b c)$ is symmetrical, the plane is a null-plane. In the nullsystem (1), let the point $\left(X_{1}, X_{2}\right.$, etc.) describe the plane $(a b c)$. Then it appears at once that the $\infty^{2}$ corresponding $M_{4}^{1}$ 's intersect in a plane whose symbol is the conjugate of $(a b c)$. Such planes we may call conjugate planes in the null-system. Clearly, a null-plane is self-conjugate.

In the null-system (4), to every point corresponds a linear $M_{4}$. Dually, to a given linear $M_{4}$ will correspond a point, the null-point of the $M_{4}$. This fact leads to the determination of the locus of the null-point of the linear tangent $M_{4}$ given by (10). To find this null-point $P_{0}\left(X_{1}^{0}, X_{2}^{0}, Y_{1}^{0}, Y_{2}^{0}, Z^{0}\right)$, we merely have to compare equation $(10)$ with the equation of the null-system

$$
Z-Z^{0}+Y_{1} X_{1}^{0}-X_{1} Y_{1}^{0}+Y_{2} X_{2}^{0}-X_{2} Y_{2}^{0}=0 .
$$

The result is readily found to be

$$
\begin{array}{r}
X_{1}^{0}=\frac{F_{Y_{1}}}{F_{Z}}, \quad X_{2}^{0}=\frac{F_{Y_{2}}}{F_{Z}^{\prime}}, \quad Y_{1}^{0}=-\frac{F_{X_{1}}}{F_{Z}^{\prime}}, \quad Y_{2}^{0}=-\frac{F_{X_{2}}}{F_{Z}^{\prime}}, \\
Z^{0}=Z+\frac{X_{1} F_{X_{1}}+X_{2} F_{X_{2}}+Y_{1} F_{Y_{1}}+Y_{2} F_{Y_{2}}}{F_{Z}} .
\end{array}
$$

When the point $P\left(X_{1}, X_{2}, Y_{1}, Y_{2}, Z\right)$ describes the locus of (9), the point $P^{0}$, which we call the conjugate point, will describe a locus, which may be named the conjugate locus. In the general case, the conjugate locus will be of four dimensions, say $F^{0}=0$. Evidently, from the definition of the conjugate locus, it is derived from the original locus by the correlation established in $R_{5}$ by the null-system. In this correlation each null-plane is invariant. Hence the pencil of null-planes tangent to $F=0$ at $P$ must have the same relation to the conjugate locus at $P^{0}$. In other words, the relation between two conjugate loci is mutual. The linear tangent $M_{4}$ and the null- $M_{4}^{1}$ at $P$ to $F=0$ are respectively 
the null- $M_{4}^{1}$ and linear tangent $M_{4}$ to $F^{0}=0$ at $P^{0}$. From the theorem on tangent null-planes at $P$, we now derive the important

Theorem. If the loci of $F=0$ and $F^{0}=0$ are conjugate under a nullsystem in $R_{5}$, and if $P$ and $P^{0}$ are corresponding points on these loci, then the $\infty^{1}$ null-planes tangent to $F=0$ at $P$ are tangent also to $F^{0}=0$ at $P^{0}$. Further, the tangent null-axes at $P$ and $P^{0}$ are identical.

Analytically, the general case arises when the tangent $M_{4}^{1}(10)$ contains four independent parameters. The null-point $P_{0}$ will then describe a four dimensional locus. If, then, the number of independent parameters in (10) is less than four, the locus of $P_{0}$ will be of three dimensions, or of two (a surface) or of one (a curve). All these cases will be covered if we consider the "envelope" of the linear $M_{4}$,

$$
E \equiv A_{1} X_{1}+A_{2} X_{2}+B_{1} Y_{1}+B_{2} Y_{2}+C Z=0,
$$

in which the coefficients are functions of certain parameters in number less than four. For example, consider the case of two parameters $u$ and $v$. Taking the first partial derivatives of $(17)$ with respect to these parameters,

$$
E_{u}=0 \text { and } E_{\mathrm{v}}=0,
$$

we see that the tangent null-axes at all the points in the plane $E=E_{u}=E_{v}=0$ pass through a common point, the null-point of $E=0$. Thus the $\infty^{4}$ tangent nullaxes form in this case $\infty^{2}$ bundles. The two other special cases mentioned are clearly those in which the tangent null-axes form $\infty^{3}$ flat pencils or $\infty^{1}$ threedimensional fascicules. In the former of these two cases, that of three parameters, the points of tangency of the axes of a pencil lie on a line.

\section{§6. Geometry of Osculating Parabolic Bands.}

The results found in the previous section are now to be interpreted in space of three dimensions by means of Lie's transformation used in $\S 4$,
(1) $X_{1}=x$,
$X_{2}=y, \quad Y_{1}=\frac{1}{2} p$,
$Y_{2}=\frac{1}{2} q$,
$Z=z-\frac{1}{2} p x-\frac{1}{2} q y$.

We obtain first some preliminary theorems. To any line in $R_{5}$ not a nullline, will correspond in $R_{3}$ a surface-element locus, for which

(a) the point-locus is a vertical parabola,

(b) the plane-locus is a parabolic cylinder.

These results follow directly as in $\S 4$, the distinction being that the condition for a null-line simply implies that the point-locus is on the plane-locus.

To any plane in $R_{5}[(2), \S 5]$ will correspond in $R_{3}$ a locus of $\infty^{2}$ elements, with the properties

(a) the point-locus is a vertical paraboloid,

(b) the plane-locus is a second vertical paraboloid. 
In fact, the plane

$$
Y_{1}=a_{1} X_{1}+a_{2} X_{2}+a_{3}, \quad Y_{2}=b_{1} X_{1}+b_{2} X_{2}+b_{3}, \quad Z=c_{1} X_{1}+c_{2} X_{2}+c_{3},
$$

gives under (1) as the point-locus,

$$
z=a_{1} x^{2}+\left(a_{2}+b_{1}\right) x y+b_{2} y^{2}+\left(a_{3}+c_{1}\right) x+\left(b_{3}+c_{2}\right) y+c_{3} \text {. }
$$

The statement (b) leads to a simple envelope problem.

The paraboloid (3) is the point-locus of $\infty^{3}$ assemblages of $\infty^{2}$ surface elements, namely, for all such that $a_{2}+b_{1}, a_{3}+c_{1}$, and $b_{3}+c_{2}$ are constant. One and only one of these assemblages is a union, namely, when $a_{2}=b_{1}, a_{3}=c_{1}$, $b_{3}=c_{2}$, and in this case, the union consists of the elements of the paraboloid. Remarks of like character apply to the plane-locus.

The theorems 1 and 2 on page 309 enable us to state these properties of the point-locus or the plane-locus: The $\infty^{2}$ surface-elements are arranged in $\infty^{1}$ parabolic bands having a common element which belongs to both point-locus and planelocus. The point-locus and the plane-locus touch on this element. Two conjugate planes in $R_{5}$ have the same point-loci and the same plane-loci.

To the null-plane

$$
Y_{1}=a_{1} X_{1}+a_{2} X_{2}+a_{3}, \quad Y_{2}=a_{2} X_{1}+b_{2} X_{2}+c_{2}, \quad Z=a_{3} X_{1}+c_{2} X_{2}+c_{3}
$$

correspond the $\infty^{2}$ surface-elements of the paraboloid

$$
z=a_{1} x^{2}+2 a_{2} x y+b_{2} y^{2}+2 a_{3} x+2 c_{2} y+c_{3} \text {. }
$$

If $a_{1} b_{2}=a_{2}^{2}$, the locus of (5) is a cylinder. That is, the $\infty^{2}$ parabolic bands of the locus have now the same plane-locus. Obviously, equation (5) gives also all planes as a special case.

The special null-planes (7) and (8) of $\S 6$ yield in $R_{3}$ the following results. The null-plane

$$
X_{\mathrm{i}}=a, \quad X_{2}=b, \quad Z=a X_{1}+b X_{2}+c,
$$

gives the $\infty^{2}$ elements of the point $(a, b, c)$. To the null-plane

$$
X_{1}=a X_{2}+b, \quad Y_{2}=c X_{2}-a Y_{1}+e, \quad Z=e X_{2}-b Y_{1}+h,
$$

correspond the $\infty^{2}$ elements of the vertical parabola *

$$
x=a y+b, \quad z=c y^{2}+2 e y+h .
$$

The discussion shows that the $\infty^{2}$ surface elements of a point or a plane, or of a vertical parabola, or of a parabolic cylinder, correspond to the points of a plane in $R_{5}$.

In the previous section it has been shown that a null-line is the axis of a pencil of null-planes. The corresponding result for space of three dimensions is this :

\footnotetext{
* Elmsland, 1. c., p. 121.
} 
$A$ parabolic band lies on $\infty^{1}$ vertical paraboloids.

The connection of these facts with the discussion of $\S 1$ is simple. The point ( $X_{1}, X_{2}$, etc.) in $R_{5}$ gives a surface-element in $R_{3}$. A consecutive point on a null-line in $R_{3}$ gives a united element. Through the point-pair in $R_{5}$ pass $\infty^{1}$ null-planes. The point $\left(X_{1}, X_{2}\right.$, etc. $)$ and each null-plane determine a curvatureelement in $R_{5}$. For clearly, in addition to $x, y, z, p, q$ determined by (1), we now have given in (5), $r=\frac{1}{2} a_{1}, 8=\frac{1}{2} a_{2}, t=\frac{1}{2} b_{2}$. Thus the $\infty^{1}$ null-surfaceelements in $R_{5}$ having a common null-axis correspond to $\infty^{1}$ curvature-elements with a common pair of united surface-elements.

The null-plane in $R_{5}$ transforms into an element union in $R_{3}$. More generally, any null-surface will transform into an element union - surface or curve.

The locus of

$$
F\left(X_{1}, X_{2}, Y_{1}, Y_{2}, Z\right)=0
$$

transforms into the partial differential equation of the first order

$$
f(x, y, z, p, q)=0 \text {. }
$$

The partial derivatives of (9) transform as follows:

$$
\begin{gathered}
F_{X_{1}}=f_{x}+\frac{1}{2} p f_{z}, \quad F_{X_{2}}=f_{y}+\frac{1}{2} q f_{z}, \quad F_{z}=f_{z}, \\
F_{Y_{1}}=2 f_{p}+x f_{z}, \quad F_{Y_{2}}=2 f_{q}+y f_{z} .
\end{gathered}
$$

Any null-surface lying entirely in (9) transforms into an integral surface of (10).

Now consider the pencil of tangent null-planes to (9) at any point, and the corresponding null-axis. Each of the $\infty^{1}$ corresponding vertical paraboloids will have in common with (10) a curvature-element, and the $\infty^{1}$ curvature elements thus arising will hang together on two united surface-elements. Hence the null-axis transforms into a parabolic band which osculates a characteristic band. This result is verified by transformation of the equations $(13), \S 5$. For by the equations of the transformation (1) and (11) we obtain immediately,

$$
\frac{d x}{2 f_{p}}=\frac{d y}{2 f_{q}}=\frac{\frac{1}{2} d p}{-f_{x}-p f_{z}}=\frac{\frac{1}{2} d q}{-f_{y}-q f_{z}}=\frac{d z}{2\left(p f_{p}+q f_{q}\right)},
$$

that is, the equations $(2)$ of $\S 3$.

We may at once write down the equations of the parabolic band osculating the characteristic band of $(10)$ at $(x, y, z, p, q)$. They are $((6), \S 4)$, since $\lambda_{1}, \lambda_{2}, \mu_{1}, \mu_{2}$ now satisfy (13) of $\S 5$,

$$
\begin{array}{ll}
x^{\prime} & =x+2 f_{p} t, \quad y^{\prime}=y+2 f_{q} t, \\
p^{\prime} & =p-2\left(f_{x}+p f_{s}\right) t, \quad q^{\prime}=q-2\left(f_{y}+q f_{s}\right) t, \\
z^{\prime} & =z+2\left(p f_{p}+q f_{q}\right) t-2\left[f_{x} f_{p}+f_{y} f_{q}+f_{s}\left(p f_{p}+q f_{q}\right)\right] t^{2} .
\end{array}
$$


The exceptional cases noted on page 308 lead to the following results.

1. If $f_{p}=f_{q}=0$, the osculating parabolic bands are hinges. The differential equation now contains no partial derivatives and its locus consists of all surfaceelements of the points of the surface $f(x, y, z)=0$.

2. If $f_{x} f_{p}+f_{y} f_{q}+f_{z}\left(p . f_{p}+q f_{q}\right)=0$ by virtue of $f=0$, the osculating bands are twisted bands. The characteristic curves are now asymptotic lines on all integral surfaces of $f(x, y, z, p, q)=0$. In fact, as explained in $\S 1$, the direction $P P^{\prime}$ of the united elements of the band is now an asymptotic direction.

3. If $f_{x}+p f_{z}=f_{y}+q f_{z}=0$, that is, if the differential equation is of the form $f(z-p x-q y, p, q)=0$, the osculating bands are flat.

Returning to 2 , we see that the fact that the osculating bands are twisted states a necessary and sufficient condition that the characteristic curves shall be asymptotic lines on all integral surfaces. In this case, Lie has shown that the point-loci of the $\infty^{4}$ bands reduce to $\infty^{3}$ lines, that is, $\infty^{1}$ bands have the same point-loci. The general question may be asked:- when do the $\infty^{4}$ osculating parabolic bands arrange themselves in $\infty^{3}$ systems of $\infty^{1}$ bands having a common point-locus? This general problem includes the case just discussed.

If we write the equations (12) in the form

then

$$
x=a y+b, \quad z=c y^{2}+2 e y+h,
$$

$$
\begin{aligned}
a \equiv f_{p} \div f_{q}, \quad b & =x-a y, \quad c=-\frac{1}{2} \frac{f_{x} f_{p}+f_{y} f_{q}+f_{z}\left(p f_{q}+q f_{q}\right)}{f_{q}^{2}}, \\
e & =\frac{1}{2}(p a+q), \quad h=z-c y^{2}-2 e y .
\end{aligned}
$$

The $\infty^{4}$ elements satisfying $f=0$ lead by these formulas to the point-loci of the osculating parabolic bands. The analytic question is, therefore, when does the elimination of $x, y, z, p, q$ from (13) and $f=0$ lead to more than one equation in the $a, b, c, e, h$ ? Without answering this question in all generality, it is not difficult to show that any one of the following is a sufficient condition :-

1. Either $a$ or $c$ constant;

2. A functional relation between $a$ and $c$.

An interesting case under 2 arises when

$$
c=k\left(1+a^{2}\right) \text {. }
$$

The $\infty^{4}$ osculating parabolic bands now lie on $\infty^{3}$ congruent vertical parabolas. The characteristic curves on all integral surfaces have the property expressed by the equation

$$
d p d x+d q d y=k\left(d x^{2}+d y^{2}\right) .
$$

Conversely, the condition $(13 b)$ is sufficient for the property of the osculating vertical bands (13a). Evidently $k=0$ gives the (ase 2 
The equations (15) of the previous section become in space of three dimensions the transformation

$$
\begin{gathered}
x^{\prime}=x+2 \frac{f_{p}}{f_{z}}, \quad y^{\prime}=y+2 \frac{f_{q}}{f_{z}}, \quad p^{\prime}=-p-2 \frac{f_{x}}{f_{z}}, \\
q^{\prime}=-q-2 \frac{f_{y}}{f_{z}}, \quad z^{\prime}=z-2 \frac{f_{p} f_{x}+f_{q} f_{y}}{f_{z}^{2}} .
\end{gathered}
$$

Given any element of the locus of $f=0$, equations (14) define an element $\left(x^{\prime}, y^{\prime}, z^{\prime}, p^{\prime}, q^{\prime}\right)$, which we call the conjugate element. Elimination of $x, y, z, p$ and $q$ from $f=0$ by means of (14) will lead to the locus of the conjugate element-called the conjugate locus. In the general case, this elimination will give a single equation

$$
f^{\prime}\left(x^{\prime}, y^{\prime}, z^{\prime}, p^{\prime}, q^{\prime}\right)=0 .
$$

The theorem of the preceding section now gives the interesting result.

Theorem. Given a partial differential equation of the first order. The $\infty^{4}$ parabolic bands which osculate the characteristic bands will in general osculate also the characteristic bands of a second partial differential equation of the first order which may be found by differentiation and elimination.

The geometric relation between conjugate equations is simply this: The elements are grouped in conjugate pairs which belong to a parabolic band osculating the characteristic bands of both equations. The given equation may, of course, be self-conjugate, that is, $f=0$ and $f^{\prime}=0$ may be identical. The equation $f f^{\prime}=0$, where $f=0$ and $f^{\prime}=0$ are conjugate, offers a case of this kind.

If the characteristic curves are asymptotic lines on all integral surfaces of $f=0$, that is, if the osculating parabolic bands are twisted bands, then the characteristics of $f^{\prime}=0$ have the same property. More generally, if the property (15) is possessed by the characteristics of $f=0$, it holds also for those of $f^{\prime}=0$.

Referring to the equations of the osculating band (12) we see that the conjugate element (14) is obtained by setting the parameter $t$ equal to $1 \div f_{z}$.

The preceding discussion assumes that $f_{z} \neq 0$, that is, that the given equation (10) contains $z$. If this is not the case and if one of the other variables $x$ or $y$ appears in the equation, say $x$, we merely need to write in (10) $p=1 \div \partial x / \partial z, q=-\partial x / \partial y \div \partial x / \partial z$, and then interchange $x$ and $z$. The case when neither $x$ nor $y$ nor $z$ appears is excluded.

The exceptions to the theorem, p. 311, are readily characterized by the aid of the results given at the close of the preceding section. Transforming (16) of that section by (1) gives an equation which may be written

$$
A_{1} x+A_{2} y+B_{1} p+B_{2} q+C\left(z-\frac{1}{2} p x-\frac{1}{2} q y\right)+D=0 .
$$


in which the coefficients $A_{1}, A_{2}$, etc., are functions of one, two or three parameters. The exceptional differential equations result, then, by forming first partial derivatives of (16) with respect to the parameters and then eliminating these, using (16). The following properties of the osculating parabolic bands characterize these differential equations.

Three parameters. The $\infty^{4}$ parabolic bands osculating the characteristic bands form $\infty^{3}$ families of $\infty^{1}$ each. The $\infty^{1}$ bands of a family osculate the characteristic bands at points lying on a vertical parabola and have a common surface-element. The latter, $\infty^{3}$ in all, constitute the conjugate locus.

Two parameters. The $\infty^{4}$ osculating parabolic bands now form $\infty^{2}$ families of $\infty^{2}$ each of this sort: the $\infty^{2}$ bands of a family have a common surfaceelement and osculate the characteristic bands at points lying on a vertical paraboloid. The conjugate locus consists of the $\infty^{2}$ common surface-elements of each family.

One parameter. The $\infty^{4}$ osculating parabolic bands are arranged in $\infty^{1}$ families of $\infty^{3}$ each such that all of one family have a common surface-element. The conjugate locus is made up of these latter elements, $\infty^{1}$ in all.

The facts here assembled follow at once from the conclusions at the end of $\S 5$ and the results established in the present section.

An interesting theorem in regard to any locus of $\infty^{2}$ surface-elements

$$
x=x(t, v), \quad y=y(t, v), \quad \ldots, \quad q=q(t, v)
$$

is obtained by the following considerations. The corresponding locus in $R_{5}$ is a surface $S$. Consider the tangent plane to this surface $S$ at a point $P$. Through $P$ and the null-point $[(5), \S 5] P^{\prime}$ of the tangent plane passes a nullline. Thus at each point of $S$ we determine a tangent null-line, and hence on $S$ a system of $\infty^{1}$ null-curves. Each null-curve corresponds to an element band satisfying (17). Hence the

Theorem. Every locus of $\infty^{2}$ surface-elements in space may be arranged in $\infty^{1}$ bands.

The locus of the null-point $P^{\prime}$ is a surface $S^{\prime}$ (if $S$ is not a developable). Moreover, it is easily seen that the osculating plane of the null-curve at $P$ is the tangent plane to $S^{\prime}$ at $P^{\prime}$. Further $P$ is the null-point of this plane. Hence the relation between $S$ and $S^{\prime}$ is mutual. The $\infty^{2}$ null-lines touching the nullcurves on $S$ touch also the null-curves on $S^{\prime}$. This result gives for space the

Theorem. Given an assemblage $A$ of $\infty^{2}$ surface-elements in space. The $\infty^{1}$ bands in which these may be arranged determine $\infty^{2}$ osculating parabolic bands which in general osculate also the $\infty^{1}$ bands of a second assemblage $A^{\prime}$ of $\infty^{2}$ surface-elements.

It appears, then, that assemblages of $\infty^{2}$ surface-elements in space are arranged in conjugate pairs, as the relation of $A$ and $A^{\prime}$ may be named. If $A$ is a union, then $A^{\prime}$ is identical with $A$. 
The equations of $A^{\prime}$ are given directly by (17) when we substitute from (17) in the right-hand members. An exception to the theorem arises when $S$ is a developable. The osculating parabolic bands are now arranged in $\infty^{1}$ families of $\infty^{1}$ each, the bands of a family having a common element and osculating the bands of the assemblage $A$ in points lying on a vertical parabola. The assemblage $A^{\prime}$ consists of a locus of $\infty^{1}$ elements.

In preparation for certain final theorems in this section we now change the surface-element coördinates as follows. Write the equation of the plane of the element thus :

$$
Z=u X+v Y \bullet-w,
$$

and take for the new coördinates of $(P, E), u, v, w, \alpha, \beta$, the latter satisfying $d v-\alpha d u-\beta d v=0$. Then we readily find the relations

$$
u=p, \quad v=q, \quad w=z-p x-q y, \quad \alpha=-x, \quad \beta=-y,
$$

between the old and new element-coördinates. The equations (12) of the parabolic band now become, if we assume that by (19),

$$
f(x, y, z, p, q)=g(u, v, w, \alpha, \beta),
$$

as follows :

$$
\begin{array}{lc}
u^{\prime}=u+2 g_{a} t, & v^{\prime}=v+2 g_{\beta} t, \\
\alpha^{\prime}=\alpha-2\left(g_{u}+\alpha g_{v}\right) t, & \beta^{\prime}=\beta-2\left(g_{v}+\beta g_{w}\right) t, \\
w^{\prime}=w+2\left(\alpha g_{a}+\beta g_{\beta}\right) t-2\left[g_{u} g_{a}+g_{v} g_{\beta}+g_{v}\left(\alpha g_{a}+\beta g_{\beta}\right)\right] t^{2} .
\end{array}
$$

The equations of the osculating parabolic band have, therefore, precisely the same form in the new coördinates. Furthermore, the same remark applies to equations (17) giving the coördinates of the conjugate element.

Let us now turn our attention to the question of trajectory-bands of a given differential equation

$$
f(x, y, z, p, q)=0 .
$$

Given a two-parameter group

$$
\begin{gathered}
x=x, \quad y=y, \quad z=z, \quad p=\phi\left(x, y, z, \bar{p}, \bar{q} ; t_{1}, t_{2}\right), \\
q=\chi\left(x, y, z, \bar{p}, \bar{q} ; t_{1}, t_{2}\right),
\end{gathered}
$$

by which the element $(P, E)$ is turned about its point, then substitution in (22) leads to the transformed equation

$$
\bar{f}\left(x, y, z, \bar{p}, \bar{q} ; t_{1}, t_{2}\right)=0,
$$

the characteristic bands of which for constant values of $t_{1}$ and $t_{2}$ may be called trajectory-bands of $f=0$. 
The case of interest in this connection is associated with the simple transformation of the above form when

$$
p=\bar{p}+t_{1}, \quad q=\bar{q}+t_{2} .
$$

Referring to the formulas (14) for the conjugate element, we easily see that the conjugate elements of $(x, y, z, p, q)$ and all its transformed elements have also a common point $\left(x^{\prime}, y^{\prime}, z^{\prime}\right)$. For the coördinates $x^{\prime}, y^{\prime}$ and $z^{\prime}$ depend upon the partial derivatives of $f=0$ and these are invariant under (24). We see further from (14) that the conjugate elements are transformed by

$$
p^{\prime}=\bar{p}^{\prime}-t_{1}, \quad q^{\prime}=\bar{q}^{\prime}-t_{2} .
$$

Hence if $f(x, y, z, p, q)=0$ and $f^{\prime}\left(x^{\prime}, y^{\prime}, z^{\prime}, p^{\prime}, q^{\prime}\right)=0$ are conjugate equations, so also are

$$
f\left(x, y, z, p+t_{1}, q+t_{2}\right)=0 \quad \text { and } \quad f^{\prime}\left(x^{\prime}, y^{\prime}, z^{\prime}, p^{\prime}-t_{1}, q^{\prime}-t_{2}\right)=0 .
$$

It may be permitted to enlarge upon the geometrical aspect of the facts just presented. Each element $(P, E)$ of $f=0$ is turned about its point $P$ by the transformation (24), and each of the $\infty^{2}$ systems of elements thus obtained is then arranged in the characteristic bands of the corresponding differential equation (23). It is to be noted that the characteristic bands of $f=0$ do not transform into those of the new equation, for (24) is not a contact transformation. If, now, we consider the osculating parabolic bands at $(P, E)$ and at each of its $\infty^{2}$ transformed elements, these bands will have in common a second point which is common to all the conjugate element

A discussion precisely similar applies when the other element coördinates $u, v, w, x_{1} \sigma$ of (19) are employed. Namely, each element is now displaced in its own plane by the transformation

$$
a=\bar{\alpha}+\sigma_{1}, \quad \beta=\bar{\beta}+\sigma_{2},
$$

the other coördinates $u, v, w$ remaining constant. Then the conjugate elements of $(u, v, w, \bar{\alpha}, \bar{\beta})$ in the transformed equation, for every $\sigma_{1}$ and $\sigma_{2}$ will also lie in a cummon plane, namely, the plane of the element $\left(u^{\prime}, v^{\prime}, w^{\prime}, \alpha^{\prime}, \beta^{\prime}\right)$ which is conjugate to the initial element $(u, v, w, \alpha, \beta)$. Furthermore, the conjugate elements satisfy the equations $\alpha^{\prime}=\bar{\alpha}^{\prime}-\sigma_{1}, \beta^{\prime}==\bar{\beta}^{\prime}-\sigma_{2}$.

The transformation (26) in the usual coördinates $(x, y, z, p, q)$ takes the form

$$
x=\bar{x}-\sigma_{1}, \quad y=\bar{y}-\sigma_{2}, \quad z=\bar{z}-p \sigma_{1}-q \sigma_{2},
$$

the coördinates $p$ and $q$ remaining constant. Evidently each element is displaced in its own plane in such a manner that the displacement parallel to the $X Y$-plane is the same for all elements.

Recapitulation of the results found may be made in the following form: 
Theorem. Let each surface-element $(P, E)(x, y, z, p, q)$ satisfying the differential equation

$$
f(x, y, z, p, q)=0,
$$

be turned about its point in such a manner that it becomes the element $\left(P, E^{\prime}\right)$ $\left(x, y, z, p-t_{1}, q-t_{2}\right)$, where $t_{1}$ and $t_{2}$ are arbitrary constants. Then the transformed elements satisfy the equation

$$
f\left(x, y, z, p+t_{1}, q+t_{2}\right)=0,
$$

and the osculating parabolic bands of $\left(P, E^{\prime}\right)$ for every $t_{1}$ and $t_{2}$ pass through a second common point,

$$
x^{\prime}=x+\frac{2 f_{p}}{f_{z}}, \quad y^{\prime}=y+\frac{2 f_{y}}{f_{z}}, \quad z^{\prime}=z-2 \frac{f_{p} f_{x}+f_{y} f_{z}}{f_{z^{2}}},
$$

namely, the point of the conjugate element of $(P, E)$ in (10).

Again, let each element $(P, E)$ be moved in its plane so that the transformed element is $\left(P^{\prime}, E\right)\left(x+\sigma_{1}, y+\sigma_{2}, z+p \sigma_{1}+q \sigma_{2}, p, q\right)$, where $\sigma_{1}$ and $\sigma_{2}$ are arbitrary constants. The new elements satisfy the equation

$$
f\left(x-\sigma_{1}, y-\sigma_{2}, z-p \sigma_{1}-q \sigma_{2}, p, q\right)=0 .
$$

The osculating parabolic bands of $\left(\boldsymbol{P}^{\prime}, E\right)$ for every $\sigma_{1}$ and $\sigma_{2}$ touch a second common plane, namely the plane of the conjugate element of $(P, E)$ in $(10)$.

We may if we so wish consider this theorem as establishing on the parabolic band which osculates a characteristic band at $(P, E)$ the conjugate element. Clearly, as far as the theorem is concerned, the relation of any equation to its conjugate is a mutual one, that is, the new parabolic bands introduced are the same for both equations.

Attention may be called to the fact that the $\infty^{2}$ parabolic bands issuing from the point $(x, y, z)$ which arise in the first part of the theorem, lie on $\infty^{1}$ parabolas, namely, the parabolas through $(x, y, z)$ and the point of the conjugate element. Similarly, the $\infty^{2}$ bands referred to in the second part lie on $\infty^{1}$ parabolic cylinders.

One final question may be answered. If we start with any element $(x, y, z, p, q)$ satisfying (10), the transformation of the theorem leads to $\infty^{2}$ elements at the point $(x, y, z)$. Further, each of these determines a parabolic band, that is, with each is associated a united element. Hence from the discussion of $\S 1$, since each band determines at $(x, y, z) \infty^{1}$ curvature elements, we see that from the element $(x, y, z, p, q)$ are derived $\infty^{3}$ curvature elements $(x, y, z, \bar{p}, \bar{q}, \bar{r}, \bar{s}, \bar{t})$. Also there are $\infty^{1}$ elements with the same $(x, y, z)$ satisfying (10). Hence for all points we derive a configuration of $\infty^{7}$ curvature elements. These must, of course, satisfy a single partial differential equation of the second order. Obviously, we obtain this equation by forming the first partial derivatives of (23) with respect to $t_{1}$ and $t_{2}$, and then eliminating these parameters. 
It should be said that the facts disclosed in the above theorem are easily foreseen in simple properties of the null-system in $R_{5}$.

The theorem states, for surface-element loci in space, facts entirely analogous to those established by Scheffers for the plane. The osculating parabolic bands belong also to a second system of differential equations obtained from the conjugate equation by the transformation (25).

The theorem given is stated without great difficulty in a form invariant within the group $G_{21}$ of all contact-transformations under which the system of parabolic bands remains invariant. The $\infty^{3}$ points or planes of space transform into a system $\sigma$ of $\infty^{3}$ vertical paraboloids each of which has a parabolic band in common with a fixed vertical paraboloid. Each element of space determines a $\sigma$-paraboloid to which it belongs. Then a two parameter group

$$
x=\phi\left(\bar{x}, \bar{y}, \bar{z}, \bar{p}, \bar{q}, t_{1}, t_{2}\right), \ldots, \quad q=Q\left(\bar{x}, \bar{y}, \bar{z}, \bar{p}, \bar{q}, t_{1}, t_{2}\right),
$$

exists such that each element is displaced upon the corresponding $\sigma$-paraboloid. This transformation turns any differential equation

into

$$
f(x, y, z, p, q)=0
$$

$$
F\left(\bar{x}, \bar{y}, \bar{z}, \bar{p}, \bar{q} ; t_{1}, t_{2}\right)=0 \text {. }
$$

The point now is this. If the conjugate element of $(x, y, z, p, q)$ in $f=0$ is $\left(x^{\prime}, y^{\prime}, z^{\prime}, p^{\prime}, q^{\prime}\right)$ and the corresponding paraboloids are $\sigma$ and $\sigma^{\prime}$, then the conjugate element of $\bar{x}, \bar{y}, \bar{z}, \bar{p}, \bar{q}$ in $F=0$ for every $t_{1}, t_{2}$ will lie upon $\sigma^{\prime}$.

\section{§7. Geometry of Osculating Cubic Bands.}

We now turn our attention to the second type of bands described in $\S 2$. The transformation of Lie used in the previous section is here replaced by the following :

$$
x=\frac{X_{1}}{Y_{1}}, \quad y=\frac{X_{2}}{Y_{2}}, \quad z=Z, \quad p=Y_{1}^{2}, \quad q=Y_{2}^{2},
$$

by which we pass from ordinary space to space of five dimensions $R_{5}$. Moreover we verify immediately,

$$
d z-p d x-q d y=d Z+X_{1} d Y_{1}-Y_{1} d X_{1}+X_{2} d Y_{2}-Y_{2} d X_{2},
$$

and the null-system of $\S 4$ bears the same relation as before to the geometry of cubic bands. We verify easily that the null-line in $R_{5}$.

(3) $X_{1}=X_{1}^{0}+\lambda_{1} t, X_{2}=X_{2}^{0}+\lambda_{2} t, Y_{1}=Y_{1}^{0}+\mu_{1} t, Y_{2}=Y_{2}^{0}+\mu_{2} t, Z=Z^{0}+\nu t$, wherein

$$
\nu+\mu_{1} X_{1}-\lambda_{1} Y_{1}+\mu_{2} X_{2}-\lambda_{2} Y_{2}=0,
$$

gives in $R_{3}$ a cubic band. For, substituting in (1), we obtain for the equations 
of the band corresponding to (3),

$$
\begin{aligned}
& x=x^{0}+\frac{\left(\lambda_{1}-\mu_{1} x^{0}\right) \cdot t}{\sqrt{p^{0}}+\mu_{1} t}, \quad y=y^{0}+\frac{\left(\lambda_{2}-\mu_{2} y^{0}\right) t}{\sqrt{q^{0}}+\mu_{2} t}, \\
& z=z^{0}+\left[\left(\lambda_{1}-\mu_{1} x^{0}\right) \sqrt{p^{0}}+\left(\lambda_{2}-\mu_{2} y^{0}\right) \sqrt{q^{0}}\right] t, \quad p=p^{0}+\mu_{1} t\left(2 \sqrt{p^{0}}+\mu_{1} t\right), \\
& q=q^{0}+\mu_{2} t\left(2 \sqrt{q^{0}}+\mu_{2} t\right),
\end{aligned}
$$

in which the signs of the radicals are unambiguous. Then clearly, the point locus is a cubic which passes through the points at infinity on the axes of $x, y$ and $z$. Call these points for convenience $A, B$ and $C$, respectively. The plane locus is the envelope of

$$
Z-z=p(X-x)+q(Y-y),
$$

in which $x, y, z, p, q$ have the values in (4). The result of this substitution may be written

$$
\begin{aligned}
\left(\mu_{1}^{2} X+\mu_{2}^{2} Y-\lambda_{1} \mu_{1}-\lambda_{2} \mu_{2}\right) t^{2} & +2\left[\mu_{1} \sqrt{p^{0}}\left(X-x^{0}\right)+\mu_{2} \sqrt{q^{0}}\left(Y-y^{0}\right)\right] t \\
& -Z+z^{0}+p^{0}\left(X-x_{0}\right)+q^{0}\left(Y-y^{0}\right)=0 .
\end{aligned}
$$

The envelope of (6) is a quadric cone. The band (4) has therefore the characteristic described in $\S 4$. The vertex of this cone must lie upon the point-locus, the cubic. It is readily verified that the value of the parameter $t$ which determines the vertex is

$$
t_{v}=\sqrt{p^{0} q^{0}} \frac{\left(\lambda_{1}-\mu_{1} x^{0}\right) \mu_{1}+\left(\lambda_{2}-\mu_{2} y^{0}\right) \mu_{2}}{\mu_{1} \mu_{2} \nu} .
$$

The vertex lies at the initial point $\left(x^{0}, y^{0}, z^{0}\right)$ when

$$
\left(\lambda_{1}-\mu_{1} x^{0}\right) \mu_{1}+\left(\lambda_{2}-\mu_{2} y^{0}\right) \mu_{2}=0 .
$$

The cubic band is now the one referred to at the close of $\S 4$.

Pass next to the consideration of what will correspond in $R_{3}$ to the general null-plane in $R_{5}$,

$$
X_{1}=a Y_{1}+b Y_{2}+c, \quad X_{2}=b Y_{1}+d Y_{2}+e, \quad Z=-c Y_{1}-e Y_{2}+f \text {. }
$$

Eliminating $Y_{1}$ and $Y_{2}$ from (9) and the first three equations of (1) we obtain as the corresponding surface in $R_{3}$

$$
\left|\begin{array}{ccc}
a-x & b & c \\
b & d-y & e \\
-c & -e & f-z
\end{array}\right|=0 \text {. }
$$

This is the equation of a cubic surface with nodes at the fixed points $A, B$ 
and $C$. Furthermore, it is easily seen that a fourth node exists at the point $(a-b c / e, d-b e / c, f+c e / b)$.

To the special null-plane

$$
X_{1}=a Y_{1}, \quad X_{2}=b Y_{2}, \quad Z=c
$$

correspond all the elements of the point $(a, b, c)$.

Two cases of null-planes not representable in the form (9) are

$$
Y_{1}=a Y_{2}+b, \quad X_{2}=c Y_{2}-a X_{1}+e, \quad Z=-e Y_{2}+b X_{1}+h,
$$

to which will correspond in $R_{3}$ a quadric cone through the fixed points $A, B$ and $C$; and

$$
Y_{1}=b, \quad Y_{2}=c, \quad Z=b X_{1}+c X_{2}+d,
$$

which gives in $R_{3}$ the plane $z=b^{2} x+c^{2} y+d$.

It may be remarked that (12) is the same as (4), $\S 6$, if $a_{1} b_{2}=a_{2}^{2}$, a case to which attention was called at the time, while (13) is also a special case of the same equations. That is, the null-planes in $R_{5}$ which under Lie's transformation gave parabolic cylinders now go over into quadric cones. Further, the null-planes (13) in $R_{5}$ give planes in $R_{3}$ under both transformations.

To the general plane not a null-plane in $R_{5}$ will correspond in $R_{3}$ an assemblage of $\infty^{2}$ elements whose points lie upon a cubic surface having $A, B$ and $C$ as nodes, while the planes envelop a cubic with four nodes, of which the fixed points $A, B$ and $C$ constitute three. This difference in character of plane-locus and point-locus is noteworthy and is readily accounted for by considerations in $R_{5}$ which may be omitted here. The three-nodal cubic referred to is entirely general.

We may develop as in the previous section the analysis for the theory of cubic bands which osculate the characteristic bands of a given partial differential equation of the first order. To avoid the details, we limit ourselves to the statement of results.

The equations of the cubic band which osculates the characteristic band of

at $(x, y, z, p, q)$ are

$$
f(x, y, z, p, q)=0
$$

$$
\begin{aligned}
x^{\prime} & =x+\frac{2 p f_{p} t}{p-\left(f_{x}+\frac{1}{\left.p f_{z}\right) t},\right.} \quad y^{\prime}=y+\frac{2 q f_{q} t}{q-\left(f_{y}+q f_{x}\right) t}, \\
1^{\left.13^{\prime}\right) \quad} \quad z^{\prime} & =z+2\left(p f_{p}+q f_{q}\right) t, \quad p^{\prime}=\frac{\left[p-\left(f_{x}+p f_{z}\right) t\right]^{2}}{p}, \\
q^{\prime} & =\frac{\left[q-\left(f_{y}+q f_{z}\right) t\right]^{2}}{q} .
\end{aligned}
$$


The osculating cubic bands of the equation

$$
f(x, y, z, p, q)=0
$$

belong also to a second equation obtained by elimination of $x, y, z, p, q$ from (14) and

$$
\begin{gathered}
x^{\prime}=x-2 p \frac{f_{p}}{f_{x}}, \quad y^{\prime}=y-2 q \frac{f_{q}}{f_{y}}, \quad z^{\prime}=z+\frac{2 p f_{p}+2 q f_{q}}{f_{z}}, \\
p^{\prime}=\frac{1}{p} \frac{f_{x}^{2}}{f_{z}^{2}}, \quad q^{\prime}=\frac{1}{q} \frac{f_{y}^{2}}{f_{z}^{2}} .
\end{gathered}
$$

The conjugate element $\left(x^{\prime}, y^{\prime}, z^{\prime}, p^{\prime}, q^{\prime}\right)$ is given by $\left(13^{\prime}\right)$ if $t=1 \div f_{z}$.

Finally, we may state in a manner analogous to the theorem at the clos? of the preceding section the

Theorem. Let each surface element $(P, E)(x, y, z, p, q)$ of the locus of

$$
f(x, y, z, p, q)=0
$$

be turned about its point in such a manner that it becomes the element $\left(P, E^{\prime}\right)$ $\left(x, y, z, p e^{-2 t_{1}}, q e^{-2 t_{2}}\right)$, where $t_{1}$ and $t_{2}$ are arbitrary constants. Then the transformed elements satisfy the equation

$$
f\left(x, y, z, p e^{2 t_{1}}, q e^{2 t_{2}}\right)=0,
$$

and the osculating cubic bands of $\left(P, E^{\prime}\right)$ for every $t_{1}$ and $t_{2}$ pass through a second common point given by the first three equations of (15).

Again, let each element $(P, E)$ be moved in its own plane in such a manner that the transformed element $\left(P^{\prime}, E\right)$ is

$$
\left(x+\sigma_{1} / \sqrt{p}, y+\sigma_{2} / \sqrt{q}, z+\sqrt{p} \sigma_{1}+\sqrt{q} \sigma_{1}, p, q\right) ;
$$

then the new elements satisfy the equation

$$
f\left(x-\sigma_{1} / \sqrt{p}, y-\sigma_{2} / \sqrt{q}, z-\sqrt{p} \sigma_{1}-\sqrt{q} \sigma_{2}, p, q\right)=0 .
$$

Furthermore, the osculating cubic bands of $\left(P^{\prime}, E\right)$ for every $\sigma_{1}$ and $\sigma_{2}$ will touch a second plane, namely the plane of the element $x^{\prime}, y^{\prime}, z^{\prime}, p^{\prime}, q^{\prime}$ given by (15).

The statement for cubic bands of the results which correspond to other theorems in the preceding section may be omitted here.

We may regard cubic bands, projectively at least, as space analogues of circles in the plane. For the circle, as a line-element locus, is determined uniquely by two fixed points (the circular points) and two given united elements, while, as we have seen, a cubic band is determined without ambiguity if we postulate that it shall pass through three fixed points and contain two given united surface-elements. 


\section{§ 8. Concluding Theorems.}

Comparison of the transformations of $\S \S 6$ and 7 gives in space the contacttransformation

(1) $x=\frac{2 x^{\prime}}{p^{\prime}}, \quad y=\frac{2 y^{\prime}}{q^{\prime}}, \quad z=z^{\prime}-\frac{1}{2} p^{\prime} x^{\prime}-\frac{1}{2} q^{\prime} y^{\prime}, \quad p=\frac{1}{4} p^{\prime 2}, \quad q=\frac{1}{4} q^{\prime 2}$,

which has the cardinal property of transforming parabolic into cubic bands. Further properties of this transformation are readily derived from the preceding discussion.

Planes transform into planes.

Parabolic cylinders passing through the extremity of the z-axis at infinity transform into quadric cones having three fixed points $A, B, C$ in common. These points lie at infinity on the axes. Also, straight lines transform into quadric cones of this family.

Vertical paraboloids and parabolas go over into four nodal cubics, three of the nodes being $A, B, C$. In special cases, the singularities may change to two nodes and one binode, all at $A, B$ and $C$.

Elimination of $p, q, p^{\prime}, q^{\prime}$ from (1) leads to the aequatio-directrix

$$
x y z-z^{\prime} x y+y^{\prime 2} x+x^{\prime 2} y=0,
$$

from which it appears that points $\left(x^{\prime}, y^{\prime}, z^{\prime}\right)$ are transformed into cubics for which $A$ and $B$ are nodes and $C$ a binode, while points $(x, y, z)$ go over into vertical paraboloids for which the planes $x^{\prime}=0$ and $y^{\prime}=0$ are principal planes.

We may draw one conclusion from the details outlined above. From the double system of generators on a paraboloid we infer at once that for a fournodal cubic there exists a double system of quadric cones each of which will pass through three of the nodes and touch the cubic along a skew cubic. Taking the four nodes by threes in the four possible ways, we obtain eight systems of enveloping quadric cones.

ShEFFIRLD ScIENTIFIC School,

February, 1910. 